Voix et Images

voixetimages

\title{
Le Canada de Radio-Canada
}

\section{Jacques Pelletier}

Volume 20, numéro 1 (58), automne 1994

Saint-Denys Garneau

URI : https://id.erudit.org/iderudit/201148ar

DOI : https://doi.org/10.7202/201148ar

Aller au sommaire du numéro

\section{Éditeur(s)}

Université du Québec à Montréal

\section{ISSN}

0318-9201 (imprimé)

1705-933X (numérique)

Découvrir la revue

Citer cet article

Pelletier, J. (1994). Le Canada de Radio-Canada. Voix et Images, 20(1), 196-200. https://doi.org/10.7202/201148ar d'utilisation que vous pouvez consulter en ligne.

https://apropos.erudit.org/fr/usagers/politique-dutilisation/ 


\section{Le Canada de Radio-Canada}

\section{Jacques Pelletier, Université du Québec à Montréal}

Tout à fait à l'image du curieux pays qui lui confie ses mandats, la société Radio-Canada possède deux têtes, deux directions, l'une pour le Canada anglais, l'autre pour le Canada français, essentiellement le Québec, deux têtes qui, c'est le moins qu'on puisse dire, ne regardent pas tout le temps dans la même direction, tout en partageant une commune obligation de célébrer les vertus et les mérites du régime fédéral. Comment CBC et la SRC s'acquittent-elles de ce mandat, comment donnent-elles à entendre et à voir cette étrange nation, toute en nuances et en paradoxes, sinon en contradictions, que constitue notre "merveilleux" pays?

C'est la question centrale qu'aborde l'ouvrage de Greg M. Nielsen sur Le Canada de Radio-Canada ${ }^{1}$. Il s'agit d'étudier une réalité empirique nettement circonscrite - la représentation du Canada dans les médias "nationaux" - dans un cadre théorique plus ambitieux visant rien de moins qu'à éláborer une sociologie critique de l'altérité. C'est cette visée que signalent très explicitement le sous-titre du livre " Sociologie critique et dialogisme culturel " et sa dédicace à André Belleau et à Marcel Rioux et par delà, aux grandes figures de Bakhtine et d'Adorno. Cette préoccupation théorique sert de fondement à la com- 
position de l'ouvrage formé d'une première partie sur les rapports entre discours social et littérature, suivie d'une seconde, plus empirique, analysant quelques textes de la SRC et de $\mathrm{CBC}$ retenus pour leur exemplarité.

Dans la première partie, Nielsen justifie longuement son recours aux concepts-pivots de ses analyses, les concepts d'homologie, de médiation et de dialogisme "empruntés" respectivement à la lignée Lukacs-Goldmann, à l'École de Francfort et à Bakhtine. S'il se livre à un rappel intéressant des positions de Lukacs et de Goldmann, il n'utilise guère par la suite le concept d'homologie. Il lui préfère manifestement celui de médiation tel que proposé par Adorno et plus tard par Habermas; la SRC lui paraît être le lieu par lequel la "médiation" passe, circule, faisant le pont entre le mandat politique très général de contribuer à l'édification d'un sentiment national commun et les auditoires visés à qui on entend faire partager cet idéal. Radio-Canada est ainsi le lieu. d'une tension entre sa vocation d'organisation dédiée à faire la promotion de cette cause "nationale" et sa dimension d'institution littéraire possédant des règles et des normes étrangères, sinon hostiles, à ce mandat politique. Elle doit, en raison de ce double statut, constamment procéder à des aménagements, à des compromis qui ne lui rendent pas la vie facile. Nielsen retient donc de l'École de Francfort ce concept de médiation qu'il emploie un peu à la manière dont Bourdieu et certains sociocritiques utilisent le concept d'institution dont il ne fait pour sa part guère usage, ce qui est étonnant compte tenu de son objet d'étude.

La grande référence demeure toutefois ici Bakhtine à qui l'auteur emprunte la théorie générale de l'énoncé et la notion importante de carnavalisation. La conception bakhtinienne de l'énoncé sous-tend ses analyses des émissions Carte blancbe et Stage; la notion de carnavalisation lui sert de point d'appui dans sa critique politique des limites et des contradictions de la SRC/CBC. En ce sens, le rappel des propositions de Bakhtine est pleinement justifié, ce qui n'est pas toujours le cas lorsqu'il s'agit de l'École de Francfort et encore moins en ce qui concerne Lukács et Goldmann dont l'évocation semble répondre davantage à des préoccupations d'ordre "scolaire ", liées aux impératifs de la thèse à l'origine de l'ouvrage, qu'à des obligations strictement scientifiques.

Quoi qu'il en soit, $\backslash$ une fois ce rappel terminé, Nielsen se livre à une description de la situation du Canada anglais au moment où CBC est créé. Dans cette partie du pays, il semble bien y avoir un primat effectif des régions sur la Nation. L'auteur va même jusqu'à soutenir 
que la "nation" n'existe pas, est "absente" dans l'imaginaire et la vie sociale des Canadiens anglais. Ce qui présente quelque consistance, c'est la réalité régionale qui témoigne elle-même d'un éclatement, d'une fragmentation. D'où le défi de construire une nation à partir des régions et en partie contre elles, de faire naître un véritable sentiment national permettant notamment de résister à la domination politique et idéologique des Américains qui envahissent les radios privées au moment même où $\mathrm{CBC}$ se développe.

La situation du Québec est différente, s'offrant comme l'image inversée de la réalité canadienne-anglaise. La "nation" y existe pleinement, y compris dans ses hésitations et ses contradictions, et c'est la "région" qui, par comparaison, semble "absente". Le défi, ici, consistera à exprimer à travers des discours, des voix et plus tard à la télévision à travers des images, cette réalité "nationale" en profonde mutation, tiraillée entre la tradition et la nouveauté et à contribuer, par cette représentation, à sa transformation en société moderne pleinement accomplie mais dans le cadre fédéral canadien, bien entendu.

Le défi, on le voit, s'avère énorme; il s'agit, à partir de deux réalités culturelles et sociales fondamentalement différentes, de produire en quelque sorte une réalité d'ordre supérieur: la "nation canadienne" qui ne pourra être en l'occurrence que la créature hybride avec laquelle nous nous débattons toujours aujourd'hui.

Au réseau français, on se montre surtout préoccupé par la transformation de la société québécoise. L'exemple évoqué par Nielsen, celui de l'émission Carte blanche réalisée par Fernand Séguin au début des années cinquante, est à ce titre particulièrement révélateur. À travers un genre "mineur ", la comédie de mours, qui relève de ce que Bakhtine appelle le "comico-sérieux", Séguin et ses collaborateurs se livrent à une satire de la société canadienne-française, en dénonçant ironiquement les traditions dépassées et les contradictions contemporaines. En cela leur discours s'apparente au propos plus immédiatement politique des intellectuels de l'époque; à leur manière ils véhiculent, par l'humour et le rire, l'idéologie de rattrapage qui anime les opposants du régime conservateur de l'U.N. dirigée par Duplessis.

Les préoccupations de la $\mathrm{CBC}$ sont autres et cela perce très nette-' ment dans les émissions comme Stage, équivalent canadien-anglais de Carte blanche. Il ne s'agit pas ici de promouvoir le moderne contre, la tradition mais de participer à la création d'un sentiment national dépassant les cadres régionaux sans toutefois donner dans l'américanisation qui représente une menace majeure pour l'identité canadienne (anglaise). Il faut donc à la fois ouvrir les frontières régionales et en 
même temps les protéger dans la mesure où elles s'opposent, par leur existence même, à la continentalisation à l'étatsunienne.

Relevant d'un cadre organisationnel bicéphale, dotées d'un mandat unique toutefois - favoriser la fameuse "unité nationale " - les deux branches de la société d'État témoignent éloquemment, dans la réalité des pratiques, des émissions tant culturelles que d'affaires publiques, de préoccupations fort différentes elles-mêmes liées à des états de culture et de société foncièrement dissemblables. À l'image du Canada, produit hybride, hétérogène, composite, essentiellement contradictoire, la société d'État élabore des émissions culturelles et littéraires réflétant dans leur contenu et leur forme même - ici une pratique différenciée de l'humour et de l'ironie - le caractère artificiel de ce pays.

À qui s'intéresse aux médias, et plus particulièrement à l'évolution de la radio avant la Révolution tranquille, l'ouvrage de Greg M. Nielsen propose une lecture passionnante. Il rejoindra également ceux et celles qui se sentent concerné(e)s par les problématiques de l'identité et de l'altérité telles qu'elles se posent au Canada. Il devrait enfin retenir l'attention des chercheurs ouvrant dans le vaste domaine de la paralittérature; l'analyse de son statut et de son usage à Radio-Canada se montre à ce titre singulièrement instructive.

Au total, nous avons affaire à une contribution stimulante qui présente toutefois deux "irritants".

Le premier "irritant", je l'ai signalé rapidement plus haut, est lié à la genèse même de l'ouvrage qui est tiré d'une thèse de doctorat et qui en a conservé certaines contraintes, dont l'auteur - et par la suite' le lecteur! - aurait pu se dispenser. Nielsen, par exemple, semble reprendre pour l'essentiel la partie théorique de sa thèse qui représente le tiers de son livre, ce qui est beaucoup compte tenu surtout qu'une partie substantielle du rappel qu'elle contient n'est pas vraiment indispensable dans l'analyse empirique qui suit: Il introduit de même; le plus souvent en notes, de longues digressions sur la dimension méthodologique et historiographique de son propos qui, parfois intéressantes en soi, retardent l'analyse et attirent l'attention sur des points pas toujours reliés à la visée centrale de l'ouvrage.

Si ce premier "irritant" rend à l'occasion la lecture fastidieuse, le second concerne le corpus même retenu et analysé par l'auteur. Extrêmement pertinent et passionnant, ce corpus demeure un peu mince: une émission par réseau avec comme point d'appui de l'étude dans chaque cas un épisode significatif: ce n'est pas beaucoup. Le lecteur (que je suis) aurait pu "en prendre" davantage et aurait volontiers 
troqué quelques coupures dans l'exposé de l'ambition théorique et de la méthodologie contre une analyse plus approfondie d'un corpus plus étendu et diversifié.

Ces deux "irritants" n'enlèvent rien à l'intérêt de l'ouvrage, je le répète, mais ils gênent par moments la lecture et ils laissent le lecteur sur sa faim, une faim que Nielsen, c'est l'un de ses mérites, sait remarquablement susciter. Son livre suggère en effet des pistes de recherche inédites que d'autres, s'inspirant de son exemple, pourraient heureusement emprunter, apportant ainsi une contribution enrichissante, stimulante au développement des études sur l'imaginaire des sociétés canadienne et québécoise.

1. Greg M.Nielsen, Le Canada de Radio-Canada. Sociologie critique et dialogisme culturel, Toronto, Éditions du Gref, coll. "Théorie ", n 4, 1994, 202 p. 